\title{
论 文
}

\section{中国汉族人群长寿老人 TERT 基因 MNS16A 长度 多态研究}

\author{
刘立娜 ${ }^{(1 \dagger}$ ，王成业 ${ }^{(2 \dagger}$ ，逯翔 ${ }^{(1)}$ ，肖富辉 ${ }^{(1)}$ ，王华伟 ${ }^{(1)}$ ，杨利琴 ${ }^{(1)}$ ，徐良友 ${ }^{(3)}$ ，孔庆鹏 ${ }^{(1)}$ \\ (1)中国科学院昆明动物研究所, 遗传资源与进化国家重点实验室, 昆明 650223; \\ (2)中国林业科学研究院资源昆虫研究所，国家林业局资源昆虫培育与利用重点实验室，昆明 650224; \\ (3)都江堰长寿研究中心, 都江堰 611830 \\ $\dagger$ 同等贡献 \\ *联系人, E-mail: kongqp@mail.kiz.ac.cn
}

收稿日期: 2013-11-25; 接受日期: 2014-04-02; 网络版发表日期: 2014-10-22

国家重点基础研究发展计划(批准号：2013CB530802)、云南省应用基础研究重点项目(批准号: 2011FA024)和国家自然科学基金(批准号: 31100909)资助

\begin{abstract}
摘要 近期研究表明, TERT 基因中的一个串联重复长度多态 MNS16A 可以调节端粒酶的 活性. 以往研究报道, 端粒酶的活性决定端粒长度从而与个体的寿命相关, 据此提出假设: MNS16A 的多态可能通过控制端粒长度从而影响人类的寿命. 为验证该假设, 本实验室从 四川省都江㘿市收集了 446 个相互没有亲缘关系的长寿老人(年龄 $\geqslant 90$ 岁，平均年龄 (94.45 \pm 3.45$)$ 岁), 同时收集了 332 个正常个体作为对照组(年龄 22 53 岁, 平均年龄 (35.00土12.00)岁). 检测长寿组和对照组的 MNS16A 长度多态, 并将两组人群的 MNS16A 等 位基因频率和基因型分布通过卡方检验进行分析. 结果表明，在长寿组和对照组之间, MNS16A 等位基因频率和基因型分布没有显著差异, 表明 MNS16A 的长度多态在中国汉族 人群中可能对寿命长度没有显著影响.
\end{abstract}

关键词

端粒酶

长寿

MNS16A 多态

长寿老人
衰老是一个十分复杂的生理过程，包括身体机 能很多方面的变化，如细胞增殖能力的衰退、免疫力 的降低及内分泌系统的变化等 ${ }^{[1 ~ 3]}$. 在这个过程中, 遗传因素和环境因素相互作用共同促进了衰老的发 生. 值得注意的是, 遗传因素在衰老过程中起着重要 的作用, 能显著影响寿命 ${ }^{[46]}$. Mitchell 等人 ${ }^{[7]}$ 在美国 宾夕法尼亚州兰开斯特县阿米什人群中的调查研究 表明, 寿命约有 $25 \%$ 的遗传力, 这就意味着遗传因素
在人类的寿命中起着非常重要的决定作用.

端粒是处于染色体远端的一种保护性结构, 可 以防止染色体的降解、端末融合、重排及染色体的磨 损等 ${ }^{[8]}$. 细胞每一次的分裂都会导致端粒的缩短，当 端粒缩短到临界值时, 细胞就会调亡, 因此端粒的长 度决定细胞的增殖潜能 ${ }^{[9,10]}$, 而端粒长度的维持则要 靠端粒酶的活性.

端粒酶是一种逆转录酶, 它可以延长端粒末端

引用格式: 刘立娜，王成业，逯翔，等. 中国汉族人群长寿老人 TERT 基因 MNS16A 长度多态研究. 中国科学: 生命科学, 2014, 44: 1168-1172 2014, 57: 1024-1027, doi: 10.1007/s11427-014-4723-1 
的 TTAGGG 重复单元, 从而保护染色体的完整和维 持细胞的增殖潜能 ${ }^{[1,12]}$. 有趣的是, 端粒酶活性的高 低与一些动物的寿命相关, 如在研究老鼠 ${ }^{[13]}$ 和鸟 类 ${ }^{[14]}$ 的寿命中都发现了这种关联, 其机制可能就是 高活性的端粒酶能更好地延长端粒的长度, 从而有 助于寿命的延长. 此外, 在人类的研究中也表明高活 性的端粒酶可以更好地维持端粒长度, 从而有助于 预防老年病的发生并因此获得长寿 ${ }^{[15,16]}$.

端粒酶是一种全酶, 主要由一个 RNA 构件、一 个有催化活性的蛋白亚单位和一些其他的端粒酶相 关蛋白构成 ${ }^{[17]}$. 其催化亚单位 (telomerase reverse transcriptase, TERT) 是端粒酶活性的核心 ${ }^{[18]}$, 研究表 明, 编码该亚单位的 TERT 基因的表达量与端粒酶的 活性紧密相关 ${ }^{[19,20]}$.

综上所述, TERT 基因的表达影响端粒酶的活性, 而端粒酶的活性又与长寿相关联, 由此推测, 影响 $T E R T$ 基因表达量的一些功能性多态位点可能通过影 响端粒酶活性从而间接与长寿相关联.

近期研究发现, 位于 TERT 基因下游(染色体位 置是 5p15.33)的一个可变串联重复多态 MNS16A, 影 响 TERT 基因的表达量及端粒酶的活性 ${ }^{[21]}$. MNS16A 所在的区域具有某种类似启动子的活性, 可以调控 $T E R T$ 的表达, 而 MNS16A 串联重复长度的变化则影 响这种类似启动子的活性; 因此, MNS16A 长度多态 的变化可通过影响 TERT 的表达从而影响端粒酶的活 性 ${ }^{[21]}$.

鉴于此，提出假设: MNS16A 不同长度的等位基 因多态可能是与长寿相关的一个遗传因素. 为验证 该假设, 本实验室在汉族人群中开展了相关性研究, 以调查 MNS16A 长度多态在长寿中可能的作用.

\section{1 研究对象与方法}

\section{1 研究对象}

从四川省都江堰市共采集 446 份长寿样本(年龄 $\geqslant 90.00$ 岁, 平均年龄 $(94.45 \pm 3.45)$ 岁 $)$. 同本课题组之 前的报道 ${ }^{[22 ~ 24]}$, 长寿老人的年龄均经过第五次人口 普查官方数据的确认, 同时还要参考长寿老人本人 的子孙代数 $(\geqslant 3)$, 及本村当地的相关纪录. 只有这 三方面的数据都吻合才能被收集作为长寿研究样本. 样本采集来源地的一些人口学方面的特征在本课题 组之前的工作中已有报道 ${ }^{[22 \sim 24]}$.
同时, 征集了当地 332 个健康但无长寿家族史的 个体作为对照组 (年龄 22 53 岁, 平均年龄 (35.00土 $12.00)$ 岁). 所有参与人员均在被告知研究内容的详 情后签署了知情同意书. 本研究遵守赫尔辛基宣言 的所有条款, 并通过了中国科学院昆明动物研究所 道德伦理委员会的审查和批准.

\section{2 基因型的检测}

长寿组以及对照组的外周血经现场采集冷冻处 理后带回实验室, 采用酚氯仿法提取总 DNA. MNS16A 多态通过 PCR 产物电泳检测, 引物的设计 参照文献[21] (正向引物: 5'-AGGATTCTGATCTCTGAAGGGTG-3'; 反向引物: 5'-TCTGCCTGAGGAAGGACGTATG-3'; GenBank 登录号: AF128894.1).

实验流程简要介绍如下: PCR 总反应体积为 20 $\mu \mathrm{L}$. 反应参数为: $95^{\circ} \mathrm{C}$ 预变性 $5 \mathrm{~min} ; 95^{\circ} \mathrm{C}$ 变性 $30 \mathrm{~s}$, $61^{\circ} \mathrm{C}$ 复性 $45 \mathrm{~s}, 72^{\circ} \mathrm{C}$ 延伸 $1 \mathrm{~min}$, 共 35 个循环; $72^{\circ} \mathrm{C}$ 后 延伸 $10 \mathrm{~min}$. PCR 产物用 $3.0 \%$ 的琼脂糖凝胶电泳检 测, 产物片段的大小用 DNA marker (TIANGEN, 中 国)对比确定. 为保证实验的准确性, $10 \%$ 的样品被抽 样进行重复检测和直接测序确认.

\section{3 统计分析}

长寿组和对照组的 MNS16A 基因型和等位基因 频率进行 Pearson's 卡方检验. 当数据中个体数小于 5 时执行 Fisher's 严格检验. $P<0.05$ 示统计显著, 然后 再进行多重比较的 Bonferroni 校正.

以上分析均采用 SPSS17.0 软件进行(SPSS Inc., Chicago, IL, USA). 长寿组和对照组的 MNS16A 基因 频率在 GENEPOP 软件中进行哈-温平衡(HWE)检 测 ${ }^{[25]}$.

\section{2 结果与讨论}

本研究检测了 446 个长寿老人和 332 个对照个体 的 MNS16A 基因型, 共观察到 3 种不同长度的等位基 因型: 243, 272 和 302 bp(图 1), 这些不同的等位基因 共有 5 种不同的基因型组合(表 1). 此外, 长寿组和对 照组的 MNS16A 等位基因频率均没有偏离哈-温平衡 $(P>0.05$, 以卡方检验及 GENEPOP 中的马尔科夫链 两种方法检验), 这与本课题组之前的研究结果相一 致 ${ }^{[22 \sim 24]}$. 


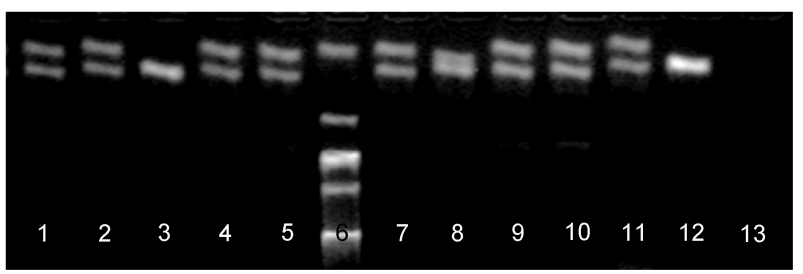

图 1 MNS16A 基因型检测

不同长度的 MNS16A 等位基因的命名基于 PCR 产物的片段大小. 不同等位基因组合而成的基因型如电泳图谱所示, 第 1 2, 4, 5, 7, $9 \sim 11$ 道是 302/243, 第 3 和 12 道是(302/302); 第 6 道是 DNA Marker; 第 8 道是 302/272; 第 13 道是阴性对照

表 1 长寿组和对照组中 MNS16A 等位基因频率、基因型分 布及纯合杂合基因型的比例

\begin{tabular}{crrrrr}
\hline & \multicolumn{3}{c}{ 长寿老人 } & \multicolumn{2}{c}{ 对照个体 } \\
\hline 基因型 & \multicolumn{2}{c}{$(N=446)$} & \multicolumn{2}{c}{$(N=332)$} & \multicolumn{1}{c}{$P$} \\
$302 / 302$ & 397 & $89.01 \%$ & 293 & $88.25 \%$ & 0.74 \\
$302 / 272$ & 18 & $4.04 \%$ & 17 & $5.12 \%$ & 0.47 \\
$302 / 243$ & 29 & $6.50 \%$ & 19 & $5.72 \%$ & 0.65 \\
$272 / 243$ & 1 & $0.22 \%$ & 1 & $0.30 \%$ & 0.83 \\
$243 / 243$ & 1 & $0.22 \%$ & 2 & $0.60 \%$ & 0.40 \\
纯合基因型 & 398 & $89.24 \%$ & 295 & $88.86 \%$ & 0.87 \\
杂合基因型 & 48 & $10.76 \%$ & 37 & $11.14 \%$ & 0.87 \\
等位基因 & & & & & \\
243 & 32 & $3.59 \%$ & 24 & $3.61 \%$ & 0.98 \\
272 & 19 & $2.13 \%$ & 18 & $2.71 \%$ & 0.46 \\
302 & 841 & $94.28 \%$ & 622 & $93.67 \%$ & 0.62 \\
\hline
\end{tabular}

在其他相关研究中曾出现过 $333 \mathrm{bp}$ 的等位基 因 $^{[21,26]}$, 而本研究的 778 个样本中(长寿组+对照组) 没有观察到 $333 \mathrm{bp}$ 的等位基因, 这可能是该等位基
因在人类群体中的频率本身就很低的缘故 ${ }^{[21]}$. 事实 上, 在另一项针对中国人群开展的研究中也发现 333 $\mathrm{bp}$ 的等位基因的频率很低，在 798 个病例中只检测到 $0.06 \%$ 的 $333 \mathrm{bp}$ 的等位基因，而在 1019 个对照个体则 没有检测到 $333 \mathrm{bp}$ 的等位基因 ${ }^{[27]}$.

MNS16A 等位基因的频率及纯合基因型、杂合 基因型的比例均列于表 1 中. 在本研究的样本中最常 见的基因型是 302/302, 随后依次是 302/243, 302/272, $272 / 243$ 及 $243 / 243$. 在长寿组中最高频率的等位基因 $302 \mathrm{bp}$ 所占的比例为 $94.28 \%$, 与对照组的 $302 \mathrm{bp}$ 出 现频率很相近(93.67\%).

长寿组与对照组的 MNS16A 等位基因频率和基 因型频率经卡方检验比较分析发现，两组之间的纯 合子比例、等位基因频率及基因型分布均无显著差异 $(P>0.05$, 表 1). 为了进一步确认性别差异是否会对 样本间的比较带来影响, 将长寿组分为男性长寿组 和女性长寿组; 对照组也相应分为男性对照组和女 性对照组. 然后再将相同性别的长寿组和对照组两 两进行比较. 分组后各组的等位基因频率经检验仍 符合哈-温平衡. 经卡方检验, 按性别分组后长寿组 和对照组之间的等位基因频率和基因型分布仍无显 著差异 $(P>0.05$, 表 2$)$.

如今, TERT 基因在调节端粒酶活性方面的重要 作用已众所周知，最近发现和报道的 TERT 基因下游 的 MNS16A 多态可通过串联重复单元长度的变化来 调节端粒酶的活性 ${ }^{[21]}$, 并且有研究发现, 不同长度的 MNS16A 和一些肿瘤的易感性相关 ${ }^{[21,27 ~ 29]}$. 然而, 衰 老是个非常复杂的生理过程, 端粒酶在衰老过程中 所起的作用可能与在肿瘤发生过程中所起的作用不

表 2 长寿组和对照组按性别分组后 MNS16A 等位基因频率、基因型分布及纯合杂合基因型的比例

\begin{tabular}{|c|c|c|c|c|c|c|c|c|c|c|}
\hline & \multicolumn{2}{|c|}{ 女性长寿老人 } & \multicolumn{2}{|c|}{ 女性对照个体 } & & \multicolumn{2}{|c|}{ 男性长寿老人 } & \multicolumn{2}{|c|}{ 男性对照个体 } & \multirow[b]{2}{*}{$P$} \\
\hline 基因型 & & & & & $P$ & & & & & \\
\hline $302 / 302$ & 288 & $89.44 \%$ & 137 & $91.95 \%$ & 0.28 & 109 & $87.90 \%$ & 156 & $85.25 \%$ & 0.51 \\
\hline $302 / 272$ & 12 & $3.73 \%$ & 6 & $4.03 \%$ & 0.87 & 6 & $4.84 \%$ & 11 & $6.01 \%$ & 0.66 \\
\hline $302 / 243$ & 21 & $6.52 \%$ & 5 & $3.36 \%$ & 0.16 & 8 & $6.45 \%$ & 14 & $7.65 \%$ & 0.69 \\
\hline $272 / 243$ & 0 & $0.00 \%$ & 1 & $0.67 \%$ & 0.14 & 1 & $0.81 \%$ & 0 & $0.00 \%$ & 0.22 \\
\hline $243 / 243$ & 1 & $0.31 \%$ & 0 & $0.00 \%$ & 0.50 & 0 & $0.00 \%$ & 2 & $1.09 \%$ & 0.24 \\
\hline 纯合基因型 & 289 & $89.75 \%$ & 137 & $91.95 \%$ & 0.45 & 109 & $87.90 \%$ & 158 & $86.34 \%$ & 0.69 \\
\hline $\begin{array}{l}\text { 杂合基因型 } \\
\text { 等位基因 }\end{array}$ & 33 & $10.25 \%$ & 12 & $8.05 \%$ & 0.45 & 15 & $12.10 \%$ & 25 & $13.66 \%$ & 0.69 \\
\hline 243 & 23 & $3.57 \%$ & 6 & $2.01 \%$ & 0.20 & 9 & $3.63 \%$ & 18 & $4.92 \%$ & 0.44 \\
\hline 272 & 12 & $1.86 \%$ & 7 & $2.35 \%$ & 0.62 & 7 & $2.82 \%$ & 11 & $3.01 \%$ & 0.89 \\
\hline 302 & 609 & $94.57 \%$ & 285 & $95.64 \%$ & 0.49 & 232 & $93.55 \%$ & 337 & $92.08 \%$ & 0.49 \\
\hline
\end{tabular}


同. 因此, 虽然有研究发现 MNS16A 长度多态和某 些肿瘤相关, 但在长寿研究中却没有发现相关性也 不足为奇.

在本研究中总样本量达到了 778 个, 但没有检测 到 MNS16A 长度变异与长寿的相关性. 一个原因可 能是 778 个样本量仍不够大, 需要更大的样本量才能
检测出这种相关性. 而另一个原因可能是 MNS16A 长度变异确实对衰老的影响很小, 不可能检测到其 与长寿之间显著的相关性. 为了进一步了解端粒酶 在人类寿命中所起的作用, 将来的研究会更多地放 在 TERT 和 TERC 这两个能明显决定端粒酶活性的基 因上.

感谢参与本研究项目的所有长寿老人和实验对照人员; 感谢都江堰当地政府和相关村委会在数据调查方面 所提供的大力协助.

\section{参考文献}

1 Takahashi Y, Kuro O M, Ishikawa F. Aging mechanisms. Proc Natl Acad Sci USA, 2000, 97: 12407-12408

2 Alonso-Fernandez P, De la Fuente M. Role of the immune system in aging and longevity. Curr Aging Sci, 2011, 4: 78-100

3 Noth R H, Mazzaferri E L. Age and the endocrine system. Clin Geriatr Med, 1985, 1: 223-250

4 Balistreri C R, Candore G, Accardi G, et al. Genetics of longevity. Data from the studies on Sicilian centenarians. Immun Ageing, 2012, 9: 8

5 Yuan R, Flurkey K, Meng Q, et al. Genetic regulation of life span, metabolism, and body weight in Pohn, a new wild-derived mouse strain. J Gerontol A Biol Sci Med Sci, 2012, 68: 27-35

6 Mockett R J, Cockrell J C, Puri S, et al. Long-lived genotypes for studies of life extension in Drosophila melanogaster. Mech Ageing Dev, 2012, 133: 359-367

7 Mitchell B D, Hsueh W C, King T M, et al. Heritability of life span in the Old Order Amish. Am J Med Genet, 2001, 102: 346-352

8 Bollmann F M. The many faces of telomerase: emerging extratelomeric effects. Bioessays, 2008, 30: 728-732

9 Aragona M, Maisano R, Panetta S, et al. Telomere length maintenance in aging and carcinogenesis. Int J Oncol, 2000, 17: 981-989

10 Lansdorp P M. Telomere length and proliferation potential of hematopoietic stem cells. J Cell Sci, 1995 , 108 (Pt 1): 1-6

11 Ahmed A, Tollefsbol T. Telomeres and telomerase: basic science implications for aging. J Am Geriatr Soc, 2001, 49: 1105-1109

12 Shen J B, Tang J Y, Zhao J C, et al. Telomerase activity and its correlation with the proliferative potential of bone marrow in aplastic anemia in children. Acta Haematol, 2002, 107: 208-212

13 Bernardes de Jesus B, Vera E, Schneeberger K, et al. Telomerase gene therapy in adult and old mice delays aging and increases longevity without increasing cancer. EMBO Mol Med, 2012, 4: 691-704

14 Haussmann M F, Winkler D W, Huntington C E, et al. Telomerase activity is maintained throughout the lifespan of long-lived birds. Exp Gerontol, 2007, 42: 610-618

15 Atzmon G, Cho M, Cawthon R M, et al. Evolution in health and medicine Sackler colloquium: Genetic variation in human telomerase is associated with telomere length in Ashkenazi centenarians. Proc Natl Acad Sci USA, 2010, 107 Suppl 1: 1710-1717

16 Houben J M, Giltay E J, Rius-Ottenheim N, et al. Telomere length and mortality in elderly men: the Zutphen Elderly Study. J Gerontol A Biol Sci Med Sci, 2011, 66: 38-44

17 Feng J, Funk W D, Wang S S, et al. The RNA component of human telomerase. Science, 1995, 269: 1236-1241

18 Deng Y, Chang S. Role of telomeres and telomerase in genomic instability, senescence and cancer. Lab Invest, 2007, 87: 1071-1076

19 Meyerson M, Counter C M, Eaton E N, et al. hEST2, the putative human telomerase catalytic subunit gene, is up-regulated in tumor cells and during immortalization. Cell, 1997, 90: 785-795

20 Nakamura T M, Morin G B, Chapman K B, et al. Telomerase catalytic subunit homologs from fission yeast and human. Science, 1997, 277: 955-959

21 Wang L, Soria J C, Chang Y S, et al. Association of a functional tandem repeats in the downstream of human telomerase gene and lung cancer. Oncogene, 2003, 22: 7123-7129

22 Li H, Wang C Y, Wang J X, et al. The neck-region polymorphism of DC-SIGNR in peri-centenarian from Han Chinese population. BMC Med Genet, 2009, 10: 134

23 Xie L, Gong Y Y, Lian S G, et al. Absence of association between SNPs in the promoter region of the insulin-like growth factor 1 (IGF-1) gene and longevity in the Han Chinese population. Exp Gerontol, 2008, 43: 962-965 
24 Xie L, Gong Y Y, Lian S G, et al. A microsatellite polymorphism in IGF1 gene promoter and longevity in a Han Chinese population. BMC Res Notes, 2010, 3: 55

25 Rousset F. genepop'007: a complete re-implementation of the genepop software for Windows and Linux. Mol Ecol Resour, 2008, 8: $103-106$

26 Wang Y, Hu Z, Liang J, et al. A tandem repeat of human telomerase reverse transcriptase (hTERT) and risk of breast cancer development and metastasis in Chinese women. Carcinogenesis, 2008, 29: 1197-1201

27 Zhang Y, Zhang H, Zhai Y, et al. A functional tandem-repeats polymorphism in the downstream of TERT is associated with the risk of nasopharyngeal carcinoma in Chinese population. BMC Med, 2011, 9: 106

28 Hofer P, Baierl A, Feik E, et al. MNS16A tandem repeats minisatellite of human telomerase gene: a risk factor for colorectal cancer. Carcinogenesis, 2011, 32: 866-871

29 Wang L, Wang L E, Mao L, et al. A functional variant of tandem repeats in human telomerase gene was associated with survival of patients with early stages of non-small cell lung cancer. Clin Cancer Res, 2010, 16: 3779-3785 